University of Nebraska - Lincoln

DigitalCommons@University of Nebraska - Lincoln

$4-2009$

\title{
Tactile learning by a whip spider, Phrynus marginemaculatus C. L. Koch (Arachnida, Amblypygi)
}

\author{
Roger D. Santer \\ University of Limerick, Ireland, roger.santer@ul.ie \\ Eileen Hebets \\ University of Nebraska - Lincoln, ehebets2@unl.edu
}

Follow this and additional works at: https://digitalcommons.unl.edu/bioscihebets

Part of the Behavior and Ethology Commons

Santer, Roger D. and Hebets, Eileen, "Tactile learning by a whip spider, Phrynus marginemaculatus C. L. Koch (Arachnida, Amblypygi)" (2009). Eileen Hebets Publications. 47.

https://digitalcommons.unl.edu/bioscihebets/47

This Article is brought to you for free and open access by the Papers in the Biological Sciences at DigitalCommons@University of Nebraska - Lincoln. It has been accepted for inclusion in Eileen Hebets Publications by an authorized administrator of DigitalCommons@University of Nebraska - Lincoln. 
Published in Journal of Comparative Physiology A: Neuroethology, Sensory, Neural, and Behavioral Physiology 195:4 (April 2009), pp. 393-399; doi: 10.1007/s00359-009-0417-8 Copyright ( ) Springer-Verlag 2009. Used by permission.

Submitted October 27, 2008; revised January 12, 2009; accepted January 16, 2009; published online February 7, 2009.

\title{
Tactile learning by a whip spider, Phrynus marginemaculatus C. L. Koch (Arachnida, Amblypygi)
}

\author{
Roger D. Santer ${ }^{1,2}$ and Eileen A. Hebets ${ }^{1}$ \\ 1. School of Biological Sciences, University of Nebraska-Lincoln, Lincoln, NE 68588, USA \\ 2. Department of Life Sciences, Schrödinger Building, University of Limerick, Limerick, Ireland \\ Corresponding author - Roger D. Santer, email roger.santer@ul.ie
}

\begin{abstract}
The ability of animals to learn and remember underpins many behavioral actions and can be crucial for survival in certain contexts, for example in finding and recognizing a habitual refuge. The sensory cues that an animal learns in such situations are to an extent determined by its own sensory specializations. Whip spiders (Arachnida, Amblypygi) are nocturnal and possess uniquely specialized sensory systems that include elongated "antenniform" forelegs specialized for use as chemo- and mechanosensory feelers. We tested the tactile learning abilities of the whip spider Phrynus marginemaculatus in a maze learning task with two tactile cues of different texture-one associated with an accessible refuge, and the other with an inaccessible refuge. Over ten training trials, whip spiders got faster and more accurate at finding the accessible refuge. During a subsequent test trial where both refuges were inaccessible, whip spiders searched for significantly longer at the tactile cue previously associated with the accessible refuge. Using high-speed cinematography, we describe three distinct antenniform leg movements used by whip spiders during tactile examination. We discuss the potential importance of tactile learning in whip spider behavior and a possible role for their unique giant sensory neurons in accessing tactile information.
\end{abstract}

Keywords: texture discrimination, associative learning, giant neuron, orientation, mechanoreception

Abbreviations: $\mathrm{CS}=$ Conditioned stimulus; $\mathrm{fps}=$ frames per second; $\mathrm{GN}=$ Giant neuron

\section{Introduction}

Learning allows an animal to modify its behavior in accordance with current environmental conditions. This ability can be crucial for survival, for example when toxic foods must be avoided after exposure or habitual shelters and/or food sources found and returned to. Many animals that shelter or forage repeatedly in particular locations find and recognize these locations (or goals), using strategies that incorporate learned associations between the goal itself and sensory cues from permanent or reliable environmental features (e.g. Shettleworth 1998). For many species, visual and olfactory cues are of particular importance for these purposes (e.g. Dittman and Quinn 1996; Dyer 1998; Shettleworth
1998; Collett and Collett 2002). However, environmental features provide sensory cues across many modalities, and the ones learned and exploited by an animal for the guidance of its behavior will likely reflect its own sensory specializations (e.g. Dyer 1998).

Whip spiders, non-spider arachnids in the order Amblypygi, are interesting from this point of view since they are nocturnal and possess uniquely specialized sensory systems highly adapted for the reception of chemoand mechanosensory cues. Walking on only their posterior three pairs of legs, their first pair of legs ('antenniform legs') are enormously elongated (2.5× their body length in some species but much longer in others), and specialized for a sensory function. These antenniform legs are covered with many types of chemo- and 
mechanosensory sensilla (Igelmund 1987), and contain at least seven unique giant neurons that carry mechanosensory information rapidly from these sensilla to the central nervous system (Igelmund and Wendler 1991a,b; Spence and Hebets 2007). When active, whip spiders constantly probe the area around them with their antenniform legs both when walking and when stationary (regardless of illumination conditions), and the antenniform legs are considered the most important structures for spatial orientation (Weygoldt 2000).

Many whip spiders reliably inhabit particular refuges during the day and return to them after foraging at night. Individual Phrynus pseudoparoulus (de Armas and Víquez 2001) occupy particular tree crevices or buttresses at the base of trees during the day, but leave them to forage at night (Hebets 2002); individual Heterophrynus batesii and H. longicornis reside on the same tree for weeks or months, occasionally leaving the tree to forage at night but returning before dawn (Beck and Görke 1974; Weygoldt 1977). Whip spiders can also orient to their refuges when artificially displaced from them: $H$. batesii displaced up to $10 \mathrm{~m}$ from their home tree can navigate back to it, within one night for displacements <7.5 m (Beck and Görke 1974; Weygoldt 2000). It has been hypothesized that sensory cues (particularly olfactory cues) received by the antenniform legs are important in this context since removing the distal tips of a whip spider's antenniform legs prevented it from returning to its refuge after being displaced (Beck and Görke 1974; Weygoldt 2000).

Since the antenniform legs of whip spiders are specialized for the reception of mechanosensory cues as well as chemosensory ones, in this study we wanted to investigate whether the whip spider $P$. marginemaculatus C. L. Koch is capable of tactile learning. Specifically, we investigated whether this species could learn to associate a tactile proximal cue with a refuge. Individual $P$. marginemaculatus are reliably found beneath particular 'home' limestone rocks in the pine rock hammock of the Florida Keys during the day, but leave these refuges to forage at night (E. A. Hebets, unpublished). These rocks vary in size, shape, and surface texture (R. D. Santer and E. A. Hebets, personal observation). As such, tactile cues received by the antenniform legs are a potential source of information that $P$. marginemaculatus could exploit in orienting to, and recognizing its home rock, as well as for the more general guidance of behavior.

\section{Materials and methods}

We investigated whether whip spiders could learn to associate a tactile proximal cue with a refuge using aversive strong lighting conditions during the dark phase of their light cycle as motivation. We performed experiments on 12 adult $P$. marginemaculatus C. L. Koch (six males and six females) collected from Big Pine Key, FL, USA in August 2006. Whip spiders were individually housed under a reversed 12:12 hour light cycle in $100 \mathrm{~mm} \times 100 \mathrm{~mm} \times 125 \mathrm{~mm}$ plastic boxes. Humidity was maintained using water-soaked cotton wicks that also allowed access to water, and whip spiders were fed one cricket twice per week. Experiments were conducted in August 2007.

\section{Experimental arena}

Experiments were conducted in a circular plastic arena with two slits cut in the floor (Figure 1a). A darkened $85 \mathrm{~mm} \times 85 \mathrm{~mm} \times 60 \mathrm{~mm}$ plastic box was placed beneath each slit as a refuge. The arena was lit from above using a fiber optic lamp (FO-150 "Lumina," Chiu Technical Corporation, Kings Park, NY, USA) with a light guide positioned approximately $300 \mathrm{~mm}$ above each arena floor slit. This strongly illuminated the arena $[1,400$ lux, measured using a VWR Scientific dual range light meter (model 62344-944), West Chester, PA, USA], but left each refuge dark. Each refuge was marked with a tactile cue: a $100 \mathrm{~mm} \times 50 \mathrm{~mm}$ sandpaper patch surrounding the slit and a $10 \mathrm{~mm} \times 60 \mathrm{~mm}$ sandpaper wall along the side of the slit closest to the arena wall (Figure $1 b$ ). Tactile cues were made from aluminum oxide sandpaper (Norton Abrasives, Worcester, MA, USA); one tactile cue had a coarse texture (P36, average particle diameter $538 \mu \mathrm{m})$, the other a fine texture (P400, average particle diameter $35 \mu \mathrm{m})$. Tactile cues were spraypainted flat black (Quick Color Spray Enamel, Roc Sales Inc., Vernon Hills, IL, USA) to minimize visual differences between them. After painting, the experimenter could easily distinguish the different tactile cues on the basis of touch.

\section{Training trials}

During training trials, the entrance to one refuge was accessible and the other was inaccessible (the entrance to the inaccessible refuge was covered with a plastic mesh that prevented a whip spider from entering). Whip spiders were divided into two groups of six (three males and three females). For one group the accessible refuge was always associated with the coarse textured tactile cue, for the other it was always associated with the fine textured tactile cue. We refer to the tactile cue associated with the accessible refuge as CS+ (CS = conditioned stimulus), and the one associated with the inaccessible refuge as CS-. Tactile cues were randomly assigned a side of the arena for each trial to prevent side effects or external landmarks indicating refuge identity or position.

For each whip spider, ten training trials were conducted over consecutive days to mimic the frequency of nightly foraging trips (E. A. Hebets, unpublished). 
Figure 1. Arena for training and test trials. a.) The arena was an opaque circular plastic container with two refuge entrances cut in the floor. Refuge entrances were marked with coarse and fine texture tactile cues (represented by different shading). b.) Both the coarse and fine texture tactile cues were sandpaper patches surrounding a refuge entrance and a 1-cm high sandpaper wall behind it. Both tactile cues were spray-painted flat black.
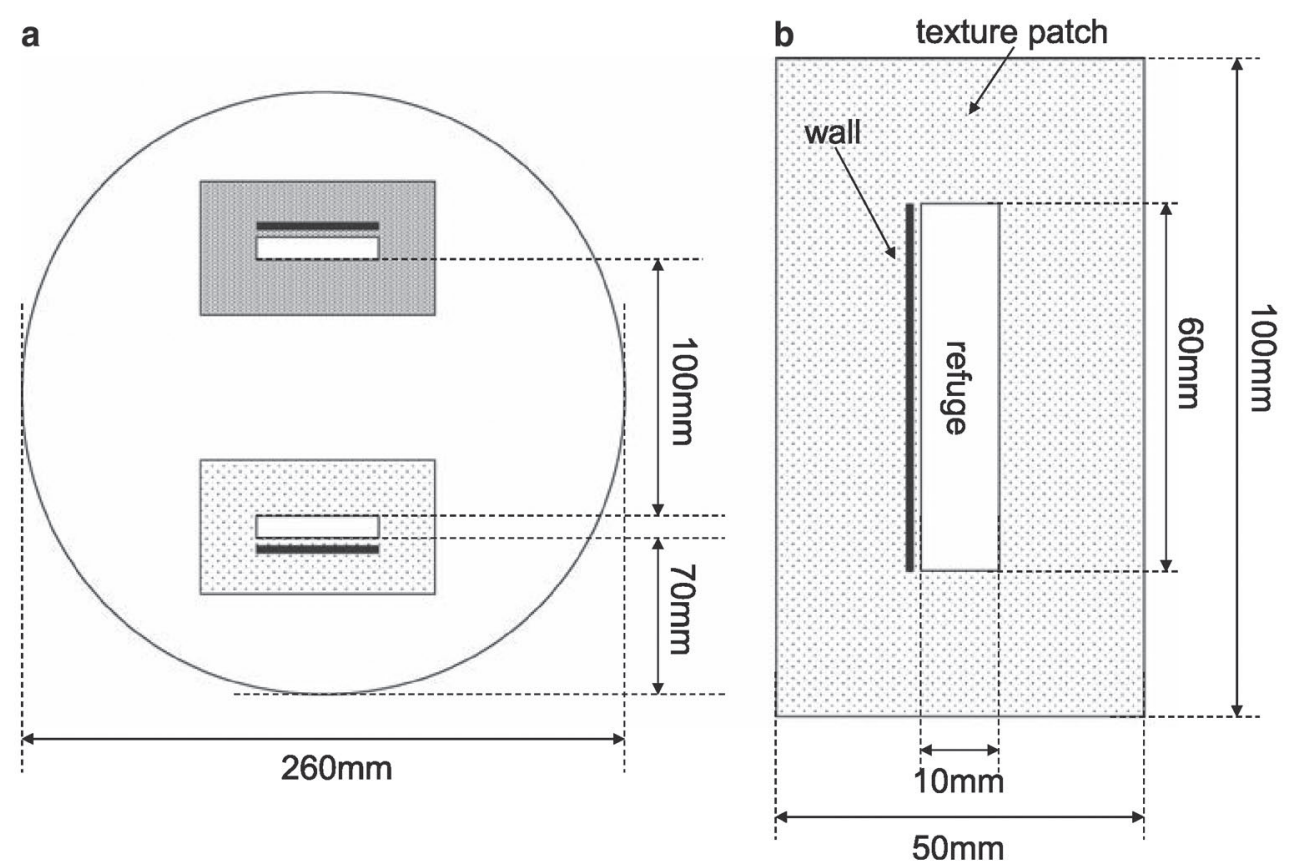

Training trials were conducted during the dark phase of the whip spiders' light cycle (their normal active phase). In each trial, whip spiders were placed in the centre of the arena under an upturned glass vial which was slowly lifted. From this position both tactile cues were within reach of the antenniform legs. Due to their nocturnal habits, whip spiders were highly motivated to escape the strong lighting of the arena by entering one of the two darkened refuges. Training trials lasted until a whip spider entered the accessible refuge associated with the CS+ tactile cue. Where this did not occur within $1 \mathrm{~h}$, an acetate screen was introduced to direct the whip spider towards CS+ and the accessible refuge, where it would eventually enter. This was usually only necessary during the first one or two training trials. After a training trial, a whip spider was returned to its normal housing under normal dark illumination. The arena, refuges, and screening were cleaned with $100 \%$ ethanol to remove chemical residues and the tactile cues were discarded (new cues were used for each training trial).

During training trials we recorded (1) the time of first movement; (2) the time taken for a whip spider to enter the accessible refuge from the start of the trial (latency); and (3) whether its first choice of refuge was correct (defined as entering the accessible refuge without having intensively explored the entrance to the inaccessible one).

During training trials, two whip spiders (a female in the fine CS+ group and a male in the coarse CS+ group) which appeared to be learning the task stopped completing it and instead remained motionless during several consecutive days of training trials. This behavior was later determined to be associated with molting and these individuals were excluded from further ex- perimentation. Ten animals remained for data analysis (three females and two males in the coarse CS+ group, and two females and three males in the fine CS+ group). However, we noted no differences between the performance of males and females, or animals in the different CS+ texture groups in our experiments.

\section{Test trials}

Test trials were carried out on the day following the last training trial. As in training trials, tactile cues were randomly assigned to an arena side, but both refuge entrances were blocked with black plastic mesh screening. Whip spiders were introduced into the centre of the arena as in training trials and observed for $45 \mathrm{~min}$. We recorded (1) the first choice of refuge (defined as the first refuge entrance intensively explored by the whip spider); (2) the amount of time a whip spider spent with any part of its body in contact with each tactile cue; and (3) the amount of time a whip spider spent actively probing a tactile cue with its antenniform legs. Time contacting and probing the tactile cues was converted to an index calculated as the fraction of total time a behavior was performed that it was directed at CS+ minus the fraction of time it was directed at CS-. Positive values indicate searches directed at CS+ and negative values indicate searches directed at CS-.

\section{High-speed cinematography}

After completion of learning experiments we filmed the antenniform leg movements of five whip spiders as they examined pieces of filter paper attached to the walls of an $85 \mathrm{~mm} \times 85 \mathrm{~mm} \times 60 \mathrm{~mm}$ plastic box. Films 
were captured at 60 or $500 \mathrm{fps}$ using a Fastcam 1024PCI high-speed digital camera (Photron USA, San Diego, CA, USA) and additional lighting was as for learning experiments.

\section{Statistical analysis of data}

Statistical analyses were carried out in SPSS v.15 for Windows (Systat Software Inc., San Jose, CA, USA) or by hand after Zar (1999). Shapiro-Wilk tests identified several cases where data differed significantly from a normal distribution, and sample sizes were relatively small. As such, we employed non-parametric statistical methods throughout.

\section{Results}

\section{General behavior}

In preliminary observations whip spiders showed little interest in either tactile cue and spent most of their time circling the wall of the arena, intensively examining it with their antenniform legs. When they encountered a tactile cue, they briefly examined it with their antenniform legs, but then directed their search effort back at the arena wall. We presume that these examinations were an attempt to find an exit to the arena and because they were not directed preferentially at the tactile cues, an innate association between these cues and a refuge location is unlikely.

\section{Training trials}

Whip spiders never entered a refuge without first examining the surrounding tactile cue with their antenniform legs. Over the course of ten training trials, latency to entering the accessible refuge decreased (Figure 2a, filled circles). There was a significant effect of training trial number on latency to accessible refuge entrance (Friedman test, $\chi^{2}=27.54, d f=9, P=0.001$ ) and there was a significant decrease in latency between training trial one and ten (paired-sample, one-tailed Wilcoxon signed ranks test, $\left.T_{-}=0, n=10, P<0.0025\right)$. Although whip spiders frequently freeze when confronted with novel or threatening stimuli (Weygoldt 2000; R. D. Santer and E. A. Hebets, personal observation), their decreasing latency to accessible refuge entrance over the training trials was not due to less time spent in freezing behavior at the start of later trials: We could not detect a difference between movement start times across training trials (Figure 2a, open circles; Friedman test, $\chi^{2}=9.12$, $d f=9, P=0.426)$, or between movement start times in training trials one and ten (paired-sample, two-tailed Wilcoxon signed ranks test, $T=19.5, n=10, P<0.50$ ). Furthermore, the number of whip spiders making a
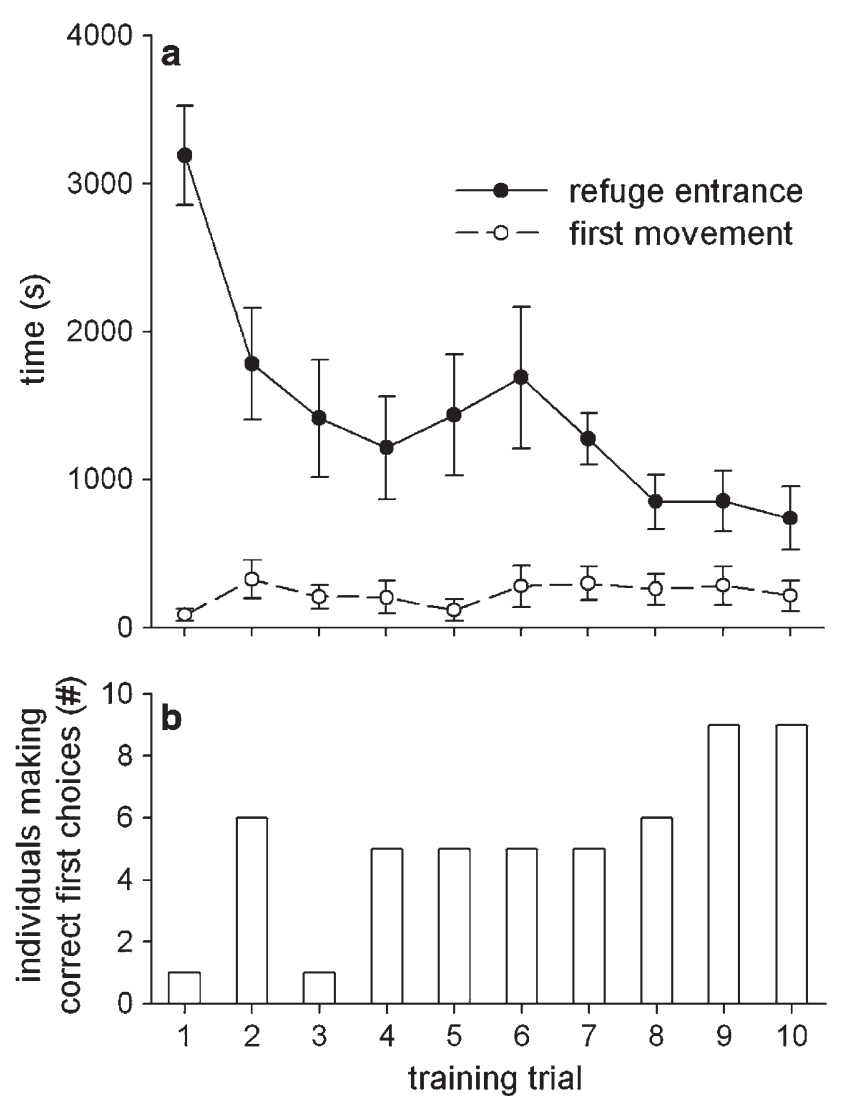

Figure 2. Evidence of tactile learning during training trials. a.) Over the ten training trials, mean latency to refuge entrance decreased (filled circles, solid line). This effect was due to a reduction in search time since there was no relationship between training trial number and the mean time that whip spiders began searching in each trial (open circles, dashed line). b.) Over ten training trials, the number of whip spiders making a correct first choice of refuge increased. Data are from ten whip spiders, for five CS+ was coarse textured, for five CS+ was fine textured. Bars are SEM.

correct first choice of refuge was correlated with training trial number, indicating increasing accuracy over the ten trials [Figure 2b; Spearman's rank correlation coefficient, $\left.\left(r_{\mathrm{s}}\right)_{\mathrm{c}}=0.732, n=10, P<0.05\right]$.

\section{Test trials}

During test trials, eight out of ten whip spiders correctly chose the CS+ refuge first, although this distribution of choices did not differ significantly from random (Chi-square goodness-of-fit test, $\chi^{2}=3.6, d f=1$, $P=0.058)$. However, whip spiders' searches were preferentially directed at the CS+ tactile cue: the indices describing (1) contact with the tactile cues, and (2) probing of the tactile cues with the antenniform legs (calculated as the fraction of time a behavior was directed at CS+ minus the fraction of time it was directed at CS-) were both significantly greater than 0 (contact index: one- 


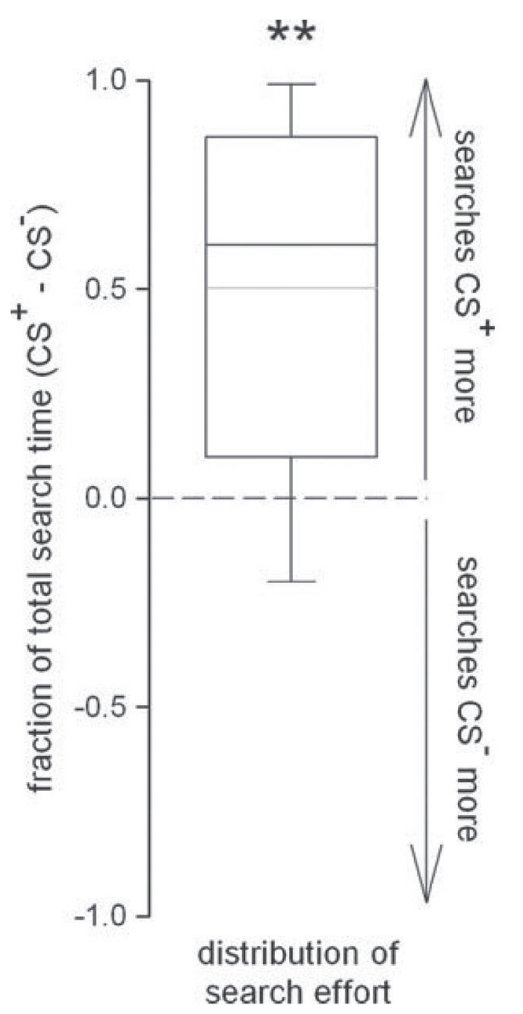

Figure 3. During test trials, whip spiders spent longer searching the CS+ tactile cue. Plot shows the fraction of search time whip spiders spent probing the positively conditioned tactile cue (CS+) with their antenniform legs, minus the fraction of time they spent probing the non-conditioned tactile cue (CS-). Positive values indicate that the majority of search effort was directed at the positively conditioned tactile cue. Box indicates the 25th and 75th percentiles, black bisecting line is the median, grey bisecting line is the mean. Whiskers indicate 5th and 95th percentiles. Asterisks indicate a significant difference $(P<0.01)$ from 0 (see text). Data are from ten whip spiders.

sample, one-tailed Wilcoxon signed ranks test against $M_{0}=0, T_{-}=10, n=10, P<0.05$; probing index: onesample, one-tailed Wilcoxon signed ranks test against $M_{0}=0, T_{-}=4, n=10, P<0.01$; Figure 3).

\section{High-speed cinematography}

Since whip spiders always examined tactile cues with their antenniform legs before trying to enter a refuge, tactile information is likely accessed using the antenniform legs. High-speed films of examination behaviors revealed three characteristic antenniform leg movements - 'tip touches', 'flat touches', and 'tip scrapes' (Figure 4). During tip touches, the antenniform leg tip was quickly tapped against the surface being examined. During flat touches, the distal half of the antenniform leg tarsus was held against the surface; and during tip scrapes the antenniform leg tip was dragged across the surface with a pronounced bend at the mid-point of the tarsus. Of 12 tip scrapes recorded, the mean length of substrate over which the antenniform leg tip was scraped was $1.55 \pm 0.27 \mathrm{~mm}$ (SEM), and the mean speed at which it was scraped was $0.018 \pm 0.003 \mathrm{~ms}^{-1}$ (SEM).

\section{Discussion}

Here we have shown that the whip spider P. marginemaculatus can learn to associate a tactile cue with a refuge. Over the course of ten training trials, the whip spiders' latency to refuge entrance decreased and the number of animals making the correct first refuge choice increased. In the test trial where both refuges were inaccessible, whip spiders directed their search effort at the tactile cue previously associated with the accessible refuge during training trials.

Tactile learning has previously been demonstrated for several other invertebrate species. The tactile properties of flower petals are used by Manduca sexta to locate nectar using its proboscis (Goyret and Raguso 2006) and can be learned by bees (Kevan and Lane 1985; Erber et al. 1998). During goal orientation, the desert ant, Cataglyphis fortis (a predominantly visual navigator), can learn tactile properties of the ground
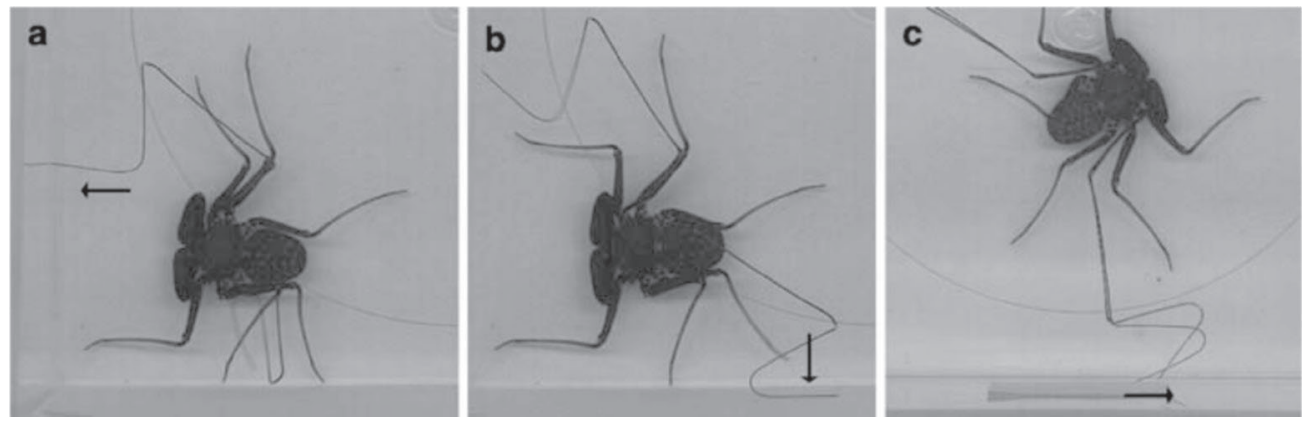

Figure 4. Antenniform leg movements during the examination of surfaces. During surface examination, three types of antenniform leg movement were evident: a.) 'Tip touches' - the tip of the antenniform leg is tapped against the surface being examined; b.) 'Flat touches' - the entire antenniform leg tarsus distal to the bend at its mid-point is held against the surface being examined; c.) 'Tip scrapes' - the tip of the antenniform leg is scraped across the surface being examined. Note the pronounced bend, proximal to articles 22/23 of the tarsus. All films were made at $60 \mathrm{fps}$. 
as navigational landmarks, and both textural and visual properties of a landmark must be in agreement for the ant to use it (Seidl and Wehner 2006). The ability of $P$. marginemaculatus to learn tactile cues and associate them with a refuge might be useful during its nocturnal orientation to, and recognition of home rocks in its natural environment. The very long antenniform legs of $P$. marginemaculatus (approximately 2.5 body lengths) would allow such tactile cues to be accessed at a distance, increasing their potential usefulness in this context (e.g. Weygoldt 2000).

In our experiments we minimized visual differences between stimuli, but we cannot completely rule out the possibility that the whip spiders could distinguish them visually (since learning was motivated by aversive illumination conditions, our experimental design did not permit experiments with blindfolded whip spiders). However, visual discrimination (or at least solely visual discrimination) is unlikely since whip spiders examined the texture patches using intensive probes of their antenniform legs, normally standing on the texture patch where the dorsal placement of their eyes would not afford close visual examination of the patches. Before entering a refuge, a whip spider always examined the surrounding texture patch with its antenniform legs, indicating that tactile examination was required for patch identification, rather than visual examination from a distance alone.

Outside of the laboratory, the nocturnal activity of $P$. marginemaculatus might be predicted to minimize the usefulness of visual cues. However, a tropical nocturnal bee and desert wandering spider have been shown to exploit visual cues for goal orientation even under extreme low light conditions (Warrant et al. 2004; Nørgaard et al. 2008). In the case of the wandering spider, relatively large lenses and a combination of temporal and spatial summation are hypothesized to allow it to salvage a coarse visual sensitivity for landscape features in its desert environment (Nørgaard et al. 2008). In these spiders, the eyes that function in nocturnal navigation have mean lens diameters of $470 \mu \mathrm{m}$ (anterior median eyes), $330 \mu \mathrm{m}$ (anterior lateral eyes), and $430 \mu \mathrm{m}$ (posterior lateral eyes), whilst the posterior median eyes (which do not function in nocturnal navigation) have lens diameters of $280 \mu \mathrm{m}$ (Nørgaard et al. 2008). By contrast, the lenses of $P$. marginemaculatus are very much smaller than those of the wandering spider-from an adult male used in this study we measured the diameter of one median eye lens as $167 \mu \mathrm{m}$, and the diameter of one lateral eye lens as $191 \mu \mathrm{m}$-indicating that they may not be as effective at capturing sufficient light for good vision at night. However, little is known about whip spider eyes and future study is necessary to establish their true visual sensitivity. Nevertheless, the uniquely adapted antenniform legs of whip spiders indicate that they play an important role in the guidance of natural behavior, regardless of whether visual cues can also be exploited (Weygoldt 2000).

A unique feature of whip spider antenniform legs is that they contain an array of giant sensory neurons with no known behavioral function (Igelmund and Wendler 1991a). Of the neurons with characterized response properties, giant interneurons GN1 and 2 respond to mechanosensory stimulation of the bristle hairs on characteristic areas of the antenniform leg tarsus, and giant sensory neurons GN6 and 7 (which are believed to be either receptor cells of a slit sense organ or putative joint receptor cells) respond to bending around a particular joint (around tarsal articles 22/23) in the mid-tarsus (Igelmund and Wendler 1991a,b; Spence and Hebets 2007). Here, high-speed cinematography revealed three movements by the antenniform legs during surface examination that appear well matched to these response properties and hint at a potential role for the giant neurons in coding textural information. Tip touches seem suited to defining the physical limits (shape) of an object and this information could be signaled by activity in GN1 which is excited by mechanical stimulation of the antenniform leg tip (Igelmund and Wendler 1991a, b). Flat touches bring a large area of the tarsus into contact with the surface being examined, and may maximize the number of contact chemoreceptive bristle hairs on the antenniform leg in contact with the surface being examined (Igelmund 1987; Foelix and Hebets 2001). The overlapping receptive fields of GN1 and 2 across the whole tarsus could be useful in positioning the antenniform leg during these movements (Igelmund and Wendler 1991a, b). During tip scrapes the antenniform leg tip is dragged across the surface being examined with a pronounced bend proximal to the joint between tarsal articles 22/23. Flexion about this joint excites GN6 and 7 (Igelmund and Wendler 1991a; Spence and Hebets 2007), and such flexions could result from the antenniform leg tip being deflected by surface irregularities during a scrape, allowing the GN6/7 response to code textural information. A similar mechanism of texture coding has been found in sensory pathways from rat whiskers (e.g. Arabzadeh et al. 2003).

Previous studies have demonstrated the olfactory sensitivity of sensilla on the antenniform legs of whip spiders and hypothesized that they play important roles in the guidance of natural behavior (Hebets and Chapman 2000; Hebets 2002). Here we have also provided the first demonstration of tactile learning in a whip spider, indicating the potential usefulness of tactile cues in the same context. Both modalities could be of considerable usefulness during activities such as finding and recognizing a habitual refuge at night. Whip spiders may prove to be excellent models for understanding the tactile guidance of behavior, but field studies are now required to investigate the true importance of these abilities in natural behavior. 
Acknowledgments - We thank the U.S. Fish and Wildlife Service and National Key Deer Refuge for permitting whip spider collection; D. P. Franklin for help conducting preliminary observations; K. A. Swoboda for animal care; D. J. Wilgers for calibrating illumination in our experimental arena; and R. M. Adams, K. D. Fowler-Finn, A. S. Rundus, S. K. Schwartz, C. A. Wei, D. J. Wilgers, and R. H. Willemart for comments on this manuscript. This work was funded by a Searle Foundation Scholars grant to E. A. Hebets. These experiments comply with the "Principles of animal care," publication No. 86-23, revised 1985 of the National Institutes of Health, and also with the current laws of the United States of America.

\section{References}

Arabzadeh E, Petersen RS, Diamond ME (2003) Encoding of whisker vibration by rat barrel cortex neurons: Implications for texture discrimination. J Neurosci 23:9146-9154

Beck L, Görke K (1974) Tagesperiodik, revierverhalten und beutefang der geisselspinne Admetus pumilio C. L. Koch im freiland. Z Tierpsychol 35:173-186

Collett TS, Collett M (2002) Memory use in insect visual navigation. Nat Rev Neurosci 3:542-552

de Armas LF, Víquez C (2001) Nueva especie de Phrynus (Amblypygi: Phrynidae) de Costa Rica. Rev Iber Aracnol 4:11-15

Dittman A, Quinn T (1996) Homing in pacific salmon: Mechanisms and ecological basis. J Exp Biol 199:83-91

Dyer FC (1998) Cognitive ecology of navigation. In: Dukas $\mathrm{R}$ (ed) Cognitive ecology: The evolutionary ecology of information processing and decision making. University of Chicago Press, Chicago

Erber J, Kierzek S, Sander E, Grandy K (1998) Tactile learning in the honeybee. J Comp Physiol A 183:737-744

Foelix RF, Hebets EA (2001) Sensory biology of whip spiders (Arachnida, Amblypygi). Andrias 15:129-140

Goyret J, Raguso RA (2006) The role of mechanosensory input in flower handling efficiency and learning by Manduca sexta. J Exp Biol 209:1585-1593

Hebets EA (2002) Relating the unique sensory system of amblypygids to the ecology and behavior of Phrynus paroulus from Costa Rica (Arachnida, Amblypygi). Can J Zool 80:286-295
Hebets EA, Chapman RF (2000) Electrophysiological studies of olfaction in the whip spider Phrynus paroulus (Arachnida, Amblypygi). J Insect Physiol 46:1441-1448

Igelmund P (1987) Morphology, sense organs and regeneration of the forelegs (whips) of the whip spider Heterophrynus elaphus (Arachnida, Amblypygi). J Morphol 193:75-89

Igelmund P, Wendler G (1991a) The giant fiber system in the forelegs (whips) of the whip spider Heterophrynus elaphus Pocock (Arachnida: Amblypygi). J Comp Physiol A 168:63-73

Igelmund P, Wendler G (1991b) Morphology and physiology of peripheral giant interneurons in the forelegs (whips) of the whip spider Heterophrynus elaphus Pocock (Arachnida: Amblygi). J Comp Physiol A 168:75-83

Kevan PG, Lane MA (1985) Flower petal microtexture is a tactile cue for bees. Proc Natl Acad Sci USA 82:4750-4752

Nørgaard T, Nilsson D-E, Henschel JR, Garm A, Wehner $R$ (2008) Vision in the nocturnal wandering spider Leucorchestris arenicola (Araneae: Sparassidae). J Exp Biol 211:816-823

Seidl T, Wehner R (2006) Visual and tactile learning of ground structures in desert ants. J Exp Biol 209:3336-3344

Shettleworth SJ (1998) Cognition, evolution, and behavior. Oxford University Press, New York

Spence AJ, Hebets EA (2007) Anatomy and physiology of giant neurons in the antenniform leg of the amblypygid Phrynus marginemaculatus. J Arachnol 34:566-577

Warrant EJ, Kelbur A, Gislén A, Greiner B, Ribi W, Wcislo WT (2004) Nocturnal vision and landmark orientation in a tropical halictid bee. Curr Biol 14:1309-1318

Weygoldt P (1977) Coexistence of two species of whip spiders (genus Heterophrynus) in the neotropical rain forest (Arachnida, Amblypygi). Oecologia 27:363-370

Weygoldt P (2000) Whip spiders (Chelicerata: Amblypygi). Their biology, morphology and systematics. Apollo Books, Stenstrup

Zar JH (1999) Biostatistical analysis, 4th edn. Prentice Hall, Upper Saddle River 